\title{
Utilización de Eucalyptus spp. Alternativas de plantaciones uruguayas para pulpa Kraft
}

\author{
Doldán, J. (1), Fariña, I. ${ }^{(1)}$, Tarigo, F. ${ }^{(1)}$ \\ Contacto: jdoldan@latu.org.uy \\ (1) Departamento de Proyectos Forestales, Laboratorio Tecnológico del Uruguay (LATU) \\ Recibido: 15/9/2008 - Aprobado: 22/12/2008
}

* Presentado en el V Congreso Iberoamericano de Investigación en Celulosa y Papel, (Guadalajara 20-23 de octubre de 2008). Guadalajara: CIADICYP. 2008.

\begin{abstract}
$\underline{\text { Resumen }}$
Las plantaciones de Eucalyptus globulus han mostrado desiguales tasas de crecimiento en diferentes regiones de Uruguay. Esto ha motivado la búsqueda de otros orígenes de semilla y especies para la producción de pulpa de celulosa. Propiedades papeleras de las pulpas blanqueadas (ECF) de especies alternativas llevan a intuir que podrían ser atractivas para mercados de pulpa de fibra corta. En este trabajo se realiza un comparativo del E. grandis, E. dunnii, E. maidenii y E. globulus (procedencia de semilla "Jeeralang") con el E. globulus predominante en Uruguay. Se discute el potencial de estas maderas como base para una mezcla en cocción, basándose en el análisis de propiedades físicas (densidad aparente básica), propiedades pulpables (rendimiento, carga de álcali activo en cocción Kraft y consumo de madera) y propiedades papeleras. En trabajos previos se han encontrado diferencias significativas entre el comportamiento pulpable del Eucalyptus globulus y Eucalyptus maidenii, sugiriendo que no sería recomendable mezclar estas especies. La misma conclusión se podría extender a las especies de Eucalyptus estudiadas. Sin embargo, teniendo en cuenta los similares requerimientos en las cargas de álcali activo, la mezcla entre especies alternativas podría ser aplicada.
\end{abstract}

Palabras clave: Eucalyptus spp., propiedades papeleras, pulpeo Kraft, rendimiento en cocción, densidad aparente básica.

\begin{abstract}
$\underline{\text { Abstract }}$
Eucalyptus globulus plantations have shown different growth rates in different sites in Uruguay. This fact has triggered the search for other pulp wood species and seed provenance. Paper making properties of ECF bleached pulps of alternative species suggest that these species could be perfectly used as hardwood bleached pulp raw materials. This study intends to compare alternative Uruguayan pulpwood species E. grandis, E. dunnii, E. maidenii and "Jeeralang" a seed provenance of E. globulus to the E. globulus most widely cultivated in the country. Physical properties of wood (Basic Density), Kraft pulping performance (pulp yield, active alkali and wood consumption) and basic papermaking properties were evaluated. Upon these results, they were considered as potential mixed base material for Kraft cooking. In previous research, significant differences were found between Eucalyptus globulus and Eucalyptus maidenii Kraft pulping behavior and no mixture was recommended. New results indicate a similar performance for each studied Eucalyptus species. However, the comparable demand in active alkali charges among them, suggests that mixtures could be successfully employed.
\end{abstract}

Key words: Eucalyptus spp., papermaking properties, Kraft pulping, cooking yield, basic density.

\section{Introducción}

Un relevamiento de las áreas forestadas a 2005 (Dirección General Forestal, MGAP, 2005) indica que el género Eucalyptus abarca aproximadamente 460.000 ha, un $50 \%$ del total implantado en el territorio uruguayo. El Eucalyptus globulus, con 270.000 ha plantadas - de las cuales un $43 \%$ se encuentra en la zona sur del país-, el Eucalyptus grandis con 160.000 ha, y otras especies como el Eucalyptus dunnii y el Eucalyptus maidenii que representan en su conjunto un $8 \%$ del total.

La fase de industrialización de la madera que está teniendo lugar en Uruguay comienza a tener efecto en la balanza comercial, provocando una tasa de crecimiento anual en exportaciones, tomada desde junio de 2004 a junio de 2007 (Rimoldi, 2007), de un total de 35,7 \%, con un incremento del $31,5 \%$ en las exportaciones de madera para pulpa. Esto se debe, principalmente desde 2005, a la aparición de plantas chipeadoras de madera que abrieron mercados como España y Japón, manteniendo entre otros a Estados Unidos, Noruega, Finlandia, Suecia y Portugal. De esa manera la madera con destino pulpable se constituye en el $72 \%$ de las exportaciones de madera. Teniendo en cuenta que la exportación de celulosa se incrementará en 1 millón de toneladas anuales producidas por BOTNIA Uruguay y el comienzo previsto de la planta de ENCE (con una producción similar) para 2010, el aporte del sector de la madera para pulpa crecerá sustancialmente.
Dada la coyuntura observada, el género Eucalyptus es y será sin dudas impulsor de investigación para encontrar los materiales primarios que más se adecuen a las necesidades de los productos finales. La búsqueda de materiales alternativos dentro de este género se comienza a dar en Uruguay debido a que el E. globulus tiene un aceptable crecimiento solamente en la zona sur-este, mientras que en zonas norte y litoral decrece sensiblemente su productividad. A esto se le agrega el problema sanitario, el cual genera pérdidas llegando en ciertos casos a incidencias de enfermedades superiores a un $20 \%$. Es en ese sentido que el conocimiento de la base forestal alternativa al $E$. globulus, tanto del E. grandis y otras especies de menor extensión como el E. dunnii y el E. maidenii, es reconocido como de vital importancia.

En el caso de la procedencia de E. globulus "jeeralang", representa una alternativa muy viable desde el punto de vista forestal, dado que genera incrementos medios anuales (IMA) de crecimiento aproximadamente $40 \%$ mayores a la media (Resquin et al. 2005). Se ha notado puntualmente un buen comportamiento en la resistencia a enfermedades del fuste (Balmelli et al. 2004) como cancros y baja incidencia de Inocutis jamaicensis (agente causal de podredumbre blanca), las cuales provocan mermas del tejido afectado y, ocasionalmente, pérdida total por quebrado del árbol antes de llegar a turnos de corte.

El objetivo primario sería obtener especímenes con alta productividad, mediante un rápido $\mathrm{y}$ sano crecimiento que genere 
madera con alta densidad y un comportamiento pulpable con el mayor rendimiento de producción de celulosa. Para lograrlo será primordial el mejoramiento genético.

En la Tabla 1 se presentan datos obtenidos en proyectos de investigación relacionados a las especies mencionadas (Resquin et al. 2005) acerca de la calidad de las fibras y las propiedades físicas y mecánicas del papel que presentan estos materiales alternativos. Las pulpas se obtuvieron en el mismo laboratorio y en las mismas condiciones que se presentan en este trabajo. Para pulpas blanqueadas con un proceso ECF estandarizado, se encuentra primero y muy notoriamente que el E. globulus revela los valores más desarrollados de índice de tracción y desgarro. No obstante, las restantes especies presentan índices de desgarro considerablemente buenos e índices de tracción dentro de los valores comerciales esperados.

\begin{tabular}{|c|c|c|c|c|c|}
\hline $\begin{array}{l}\text { Propiedades } \\
\text { Papeleras * }\end{array}$ & 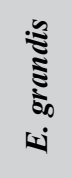 & 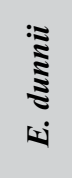 & 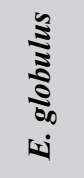 & 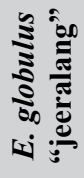 & 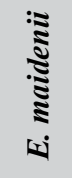 \\
\hline $\begin{array}{c}\text { Grado } \\
\text { de blanco } \\
(\% \text { ISO) } * *\end{array}$ & 87,4 & 90,9 & 89,3 & 89,0 & 90,4 \\
\hline${ }^{o} \mathrm{SR} * * *$ & 24 & 25 & 23 & 23 & 25 \\
\hline $\mathbf{N}^{0}$ Rev. *** & 1900 & 2000 & 2200 & 2200 & 2200 \\
\hline $\begin{array}{l}\text { Índice de Tracción } \\
\text { (N.m/g) } * * *\end{array}$ & 75,9 & 72,8 & 86,5 & 74,5 & 75,7 \\
\hline $\begin{array}{l}\text { Índice de Desgarro } \\
\left(\mathbf{m N} \cdot \mathrm{m}^{2} / \mathrm{g}\right) * * *\end{array}$ & 9,4 & 8,9 & 9,5 & 9,7 & 9,1 \\
\hline
\end{tabular}

Tabla 1. Propiedades papeleras para E. spp.

*Fuente (Resquin et al. 2005); **Blanqueo ECF: ODo(Ep)D1P; $* * \star$ Refinamiento PFI

Se han determinado en trabajos anteriores (Doldán, 2007) diferencias significativas en el comportamiento pulpable del $E$. globulus y E. maidenii, sugiriendo que no sería recomendable ingresar mezclas de estas dos especies a digestores industriales. En el presente informe se estudian las sinergias e incompatibilidades entre las especies de eucalipto plantadas en el territorio nacional con destino pulpable mediante el estudio de las propiedades físicas y pulpables. Se consideran para esto los siguientes parámetros de calidad: densidad aparente básica, rendimiento pulpable a Kappa 20 por proceso Kraft y consumo de madera para pulpa $\mathrm{m}^{3} / \mathrm{t}$ celulosa.

\section{Materiales y Métodos}

Materiales estudiados: La totalidad de las muestras provienen de plantaciones comerciales donde las edades de corte son, en todos los casos, entre 8 y 10 años.

- Eucalyptus globulus ssp. globulus (E. globulus)

- Eucalyptus grandis

- Eucalyptus dunnii

- Eucaliptus globulus ssp. maidenii (E. maidenii)

- Eucalyptus globulus ssp. globulus con procedencia de semilla Jeeralang (E. globulus "jeeralang")
Propiedades físicas de la madera: Densidad aparente básica (Dab) se determina según técnica LATU basada en Normas TAPPI T254 y ASTM 2395. La relación entre la productividad y rendimiento de la madera de Eucalyptus depende, entre otras propiedades, de la densidad aparente que esta posea. Por esta razón se prioriza la determinación de dicha propiedad como la más importante, por su influencia directa tanto en el rendimiento real como económico.

Digestión de chips por proceso Kraft: Cocción de chips de madera en digestores batch rotatorios. En este trabajo se han fijado la totalidad de las condiciones de cocción (Tabla 2), siendo variable únicamente el "Porcentaje de Álcali Activo" (\%AA). Este último se modifica con el cometido de alcanzar un contenido de lignina residual acorde al índice de Kappa = 20+/-1 en la pulpa resultante de cocción.

\begin{tabular}{|l|c|}
\hline Parámetro fijo & COCCIÓN \\
\hline Álcali Activo AA $\left(\mathrm{g} / \mathrm{l} \mathrm{NaO}{ }_{2}\right)$ & $120+/-10$ \\
\hline Sulfidez SZ $(\%)$ & $25+/-1$ \\
\hline Temperatura máxima $\left({ }^{\circ} \mathrm{C}\right)$ & 157 \\
\hline Tiempo subida/Tmáx (mín/mín) & $30 / 145$ \\
\hline Relación Licor/Madera & 3,5 \\
\hline Toma de chips anhidra (gr. o.d.) & 200 \\
\hline Porcentaje de Álcali Activo (\%AA) & Variable \\
\hline Equipo utilizado & $\begin{array}{c}\text { Cápsula en } \\
\text { digestor rotatorio }\end{array}$ \\
\hline
\end{tabular}

Tabla 2. Parámetros fijos y variables de cocción.

Tamizado de pasta y rendimiento de celulosa: Determinación del peso anhidro de rechazos y pulpa resultante con respecto a la muestra inicial de chips, obteniendo de esta forma el rendimiento de celulosa (rendimiento bruto y tamizado). El primero incluye los rechazos o incocidos, mientras que el segundo solamente toma en cuenta las fibras resultantes del proceso de tamizado.

$\mathbf{N}^{\circ}$ de Kappa: Tappi standard (T 236 Kappa number of pulp). El método de Kappa, permite una medida estimativa del contenido de lignina residual en pulpa. Es el consumo, en ml, de $\mathrm{KMnO}_{4} 0.1 \mathrm{~N}$ por gramo de pulpa seca. Los resultados se corrigen para un consumo de $\mathrm{KMnO}_{4}$ del $50 \%$ del total agregado.

\section{Resultados y Discusión}

Densidad Aparente Básica (Dab): Observando la Figura 1 se visualizan las distribuciones de densidades de la madera proveniente de los cinco materiales estudiados. En primer lugar, se observa a la izquierda el E. grandis, con una densidad aparente básica notoriamente menor a la de las demás especies, un promedio de $0,423 \mathrm{~g} / \mathrm{cm}^{3}$ (Tabla 3). Posteriormente, el E. dunnii presenta un promedio de 0.499 $\mathrm{g} / \mathrm{cm}^{3}$, propio de una especie con gran dispersión en los datos con un coeficiente de variación del 8,1 \% (mayor rango). 


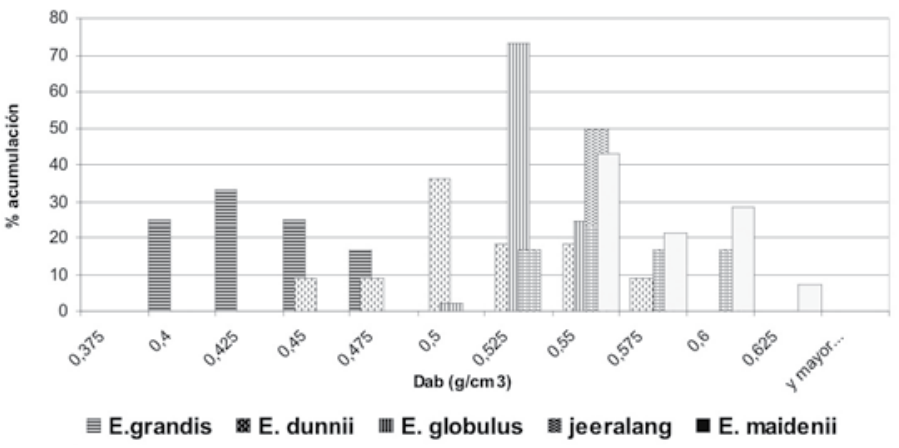

Figura 1. Histograma de densidad aparente básica para

E. spp.

El E. globulus muestra la menor dispersión con un C.V. de 1,7 \%, acumulando el $73 \%$ de los valores obtenidos en un rango entre 0,495 a $0,544 \mathrm{~g} / \mathrm{cm}^{3}\left(\right.$ media $\left.=0,520 \mathrm{~g} / \mathrm{cm}^{3}\right)$. Para la procedencia "jeeralang", las muestras estudiadas arrojan un Dab promedio de $0,550 \mathrm{~g} / \mathrm{cm}^{3}$ lo que representa un 5,6\% mayor que el E. globulus. Por último, el E. maidenii se caracteriza por incluir especímenes de una muy alta densidad, con un máximo de $0,619 \mathrm{~g} / \mathrm{cm}^{3}$ y una media de $0,559 \mathrm{~g} / \mathrm{cm}^{3}$, lo que corresponde a un $7.3 \%$ mayor al E. globulus.

\begin{tabular}{|c|c|c|c|c|c|}
\hline $\operatorname{Dab}\left(\mathrm{g} / \mathrm{cm}^{3}\right)$ & 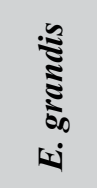 & 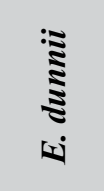 & 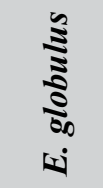 & 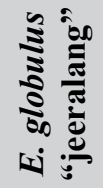 & 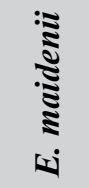 \\
\hline Media & 0,423 & 0,499 & 0,520 & $\mathbf{0 , 5 5 0}$ & 0,559 \\
\hline Desv.est. & 0,0255 & 0,0407 & 0,0086 & 0,0260 & 0,0265 \\
\hline C.V.\% & 6,0 & 8,1 & 1,7 & 4,7 & 4,7 \\
\hline Rango & 0,076 & 0,144 & 0,049 & 0,075 & 0,090 \\
\hline Mínimo & 0,390 & 0,430 & 0,495 & 0,522 & 0,529 \\
\hline Máximo & 0,466 & 0,574 & 0,544 & 0,597 & 0,619 \\
\hline
\end{tabular}

Tabla 3. Densidad aparente básica para E. spp.

Por otra parte, se ha podido comprobar que el crecimiento no correlaciona con la densidad aparente básica en todas las especies estudiadas, por lo cual se hace factible la elección de árboles con alta densidad para mejoramiento genético que generen, al mismo tiempo, altas productividades forestales.

\section{Propiedades pulpables}

Rendimiento de cocción y álcali activo: En trabajos anteriores se ha realizado un comparativo entre el E. globulus y E. maidenii, llegando a la conclusión de que existen dificultades reales para realizar una mezcla de estas dos especies en un digestor de cocción Kraft. Esto se debe especialmente a las diferentes cargas de álcali activo necesarias para alcanzar un $\mathrm{N}^{\mathrm{o}}$ de Kappa de 20.

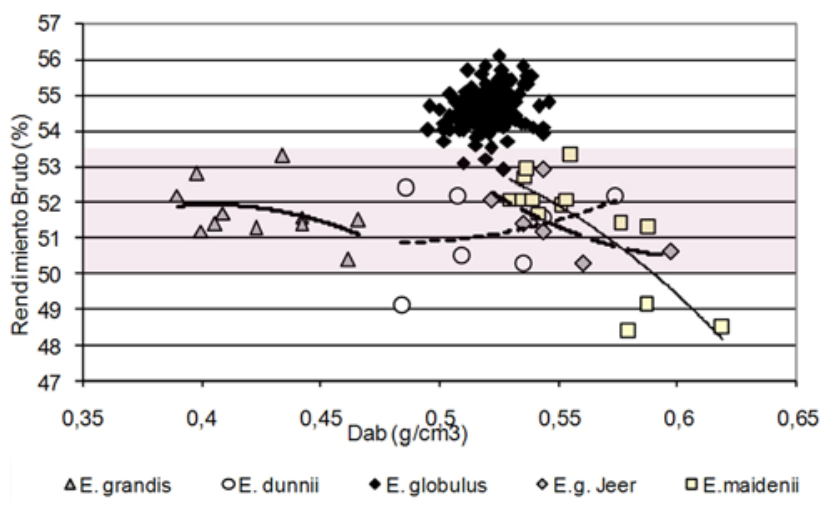

Figura 2. Rendimiento Bruto en cocción Kraft vs. densidad aparente básica para Eucalyptus spp.

En esta oportunidad se incorporan en el estudio comparativo $E$. grandis, E. dunnii y E. globulus "jeeralang", y se puede observar en una primera lectura una marcada diferencia entre el E. globulus y las demás especies (Figura 2).

E. globulus presenta nuevamente los valores más altos de rendimiento bruto de celulosa, sobresaliendo sobre los restantes. Los valores encontrados varían entre $53 \%$ y $56 \%$, siendo el valor promedio $54,5 \%$ de rendimiento en celulosa anhidra.

Para las demás especies se observa una franja de variación entre $50 \%$ y $53,5 \%$, donde los promedios son comparables tomando valores entre $51,1 \%$ y $51,7 \%$ (Tabla 4 ). Las distribuciones dentro de los rangos se mantienen en la franja salvo en el caso del E. maidenii, en el cual las características pulpables decrecen significativamente a densidades mayores a $0,570 \mathrm{~g} / \mathrm{cm}^{3}$. Esto significa que $E$. grandis, E. dunnii, E. globulus "jeeralang" y el rango hasta $0,570 \mathrm{~g} / \mathrm{cm}^{3}$ de E. maidenii responderían similarmente a procesos de cocción, alcanzando rendimientos aceptables. Se debe tener en cuenta que los materiales son comerciales y sin mejoramiento genético. Técnicas de elección y propagación vegetativa de árboles plus que tengan en cuenta la calidad del producto final podrían ser esenciales en lo que refiere al incremento de la productividad de estas especies.

\begin{tabular}{|c|c|c|c|c|c|}
\hline $\begin{array}{c}\text { Rendimiento Bruto } \\
\text { (\%) }\end{array}$ & 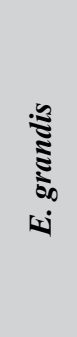 & 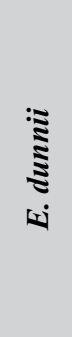 & 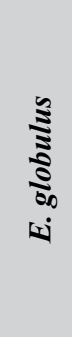 & 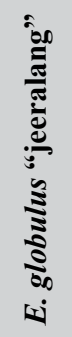 & 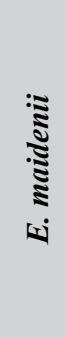 \\
\hline Media & 51,7 & 51,1 & 54,5 & 51,4 & 51,4 \\
\hline Desvest & 0,8 & 1,2 & 0,5 & 1,0 & 1,6 \\
\hline C.V.\% & 1,5 & 2,4 & 0,9 & 1,9 & 3,1 \\
\hline Rango & 2,9 & 3,3 & 3,2 & 2,6 & 4,9 \\
\hline Mínimo & 50,4 & 49,1 & 52,9 & 50,3 & 48,4 \\
\hline Máximo & 53,3 & 52,4 & 56,1 & 52,9 & 53,3 \\
\hline
\end{tabular}

Tabla 4. Rendimiento Bruto a Kappa 20 para E. spp.

Esta similitud de comportamiento es reafirmada mediante un comparativo entre los valores de carga de Álcali Activo (AA\%) utilizado en cocción para la obtención de pulpas de celulosa con un grado similar de $\mathrm{N}^{\circ}$ de Kappa $(\mathrm{K}=20)$. Se encuentra claramente en la Figura 3 una brecha entre el E. globulus y las demás sub especies, 
marcando dos o más franjas o rangos de uso. Se detecta una zona de bajo álcali entre 12.5 y $14 \%$ AA para el E. globulus (con un promedio de 13,2 \%AA), la cual respondería a una delignificación satisfactoria en cocción Kraft.

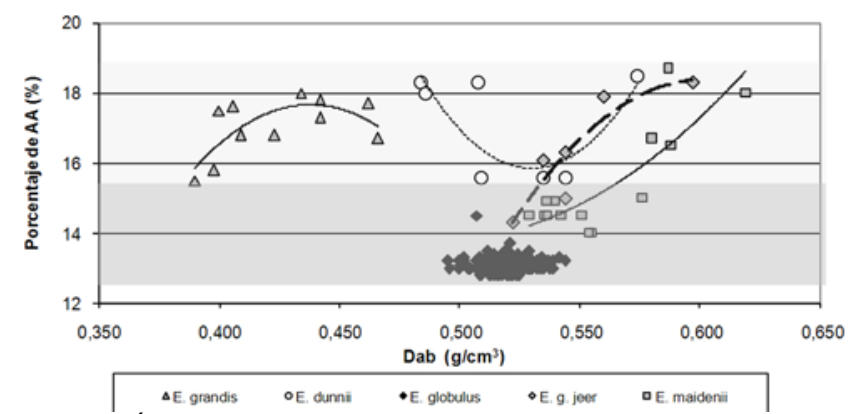

Figura 3. Álcali Activo en cocción Kraft vs. densidad aparente básica para E. spp.

Como se ha visto anteriormente, las condiciones de cocción necesarias para el E. globulus son las menos exigentes a nivel de laboratorio en cuanto a carga de químicos en licor blanco. Considerando la versatilidad de los procesos continuos o super-batch, sería posible encontrar algunas plantaciones de E. maidenii con bajas densidades que pudieran, a su vez, ser mezcladas con E. globulus con cargas algo mayores. Sin embargo, la diversidad de esta especie lleva a que pueda ser hallado un rango muy amplio de respuestas a las exigencias de \%AA, lo cual podría resultar en una práctica peligrosa, con requerimientos de hasta $18 \%$ AA. Por lo expresado anteriormente, y observando la Figura 3, se podría hacer una categorización de las maderas estudiadas en dos franjas. La primera franja sería desde 12,5 a $15,5 \% \mathrm{AA}$, enrabando en ésta al E. globulus y al rango de hasta $0,570 \mathrm{~g} / \mathrm{cm}^{3}$ de $E$. maidenii. La segunda, desde 15,5 a $19 \%$ AA, en la cual se encuentran el E. grandis, E. dunnii, E. globulus "jeeralang" y E. maidenii (mayor a $0,570 \mathrm{~g} / \mathrm{cm}^{3}$ ). Se percibe entonces la posibilidad de mezclar estas especies en cocciones Kraft, pudiéndose manejar una relativa homogeneidad en el proceso.

Consumo de madera y productividad: Al considerar la combinación de la densidad aparente básica de madera y el rendimiento de pulpa en cocción, se incorpora un parámetro sustancial para el manejo de la materia prima utilizada. En este caso se presenta el consumo de madera para generar una tonelada de pulpa seca al aire (a.d.). En la Figura 4 se puede apreciar el consumo obtenido para el rango de Dab estudiado para cada especie.

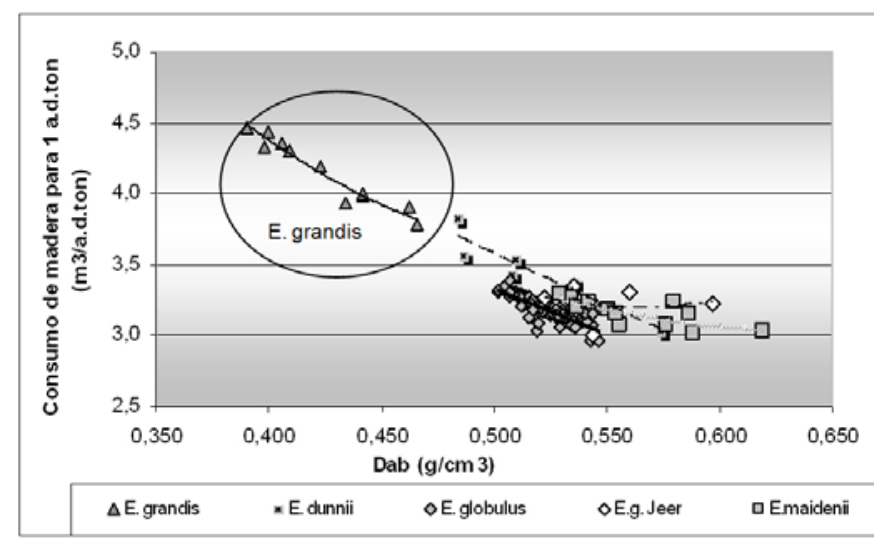

Figura 4. Consumo de madera verde $\left(\mathrm{m}^{3}\right)$ para una tonelada de pulpa marrón a Kappa 20 vs. densidad aparente básica para E. spp.

Es considerable la diferencia encontrada entre el E. grandis y los demás Eucalyptus estudiados. Esta especie presenta un promedio de consumo cercano a $4 \mathrm{~m}^{3} /$ a.d.ton, mientras que las demás se encuentran en un promedio cercano al 3,2 $\mathrm{m}^{3} /$ a.d.ton. La Dab influye directamente, produciendo un efecto inverso en este parámetro, haciéndose evidente para el E. Grandis, donde su Dab promedio es comparativamente más baja aunque el rendimiento pulpable es similar. Lo mismo se puede observar para las muestras de E. dunnii con baja densidad aparente básica $\left(<0,500 \mathrm{~g} / \mathrm{cm}^{3}\right)$, presentando consumos mayores a $3,5 \mathrm{~m}^{3} /$ a.d.ton. Al igual que lo expresado anteriormente, mediante selección de árboles plus con altos crecimientos y rendimientos pulpables, sería posible obtener consumos sustancialmente menores a $3 \mathrm{~m}^{3} /$ a.d.ton.

Haciendo el ejercicio teórico de combinar de a tres las especies en mezclas de composición de partes iguales, se pueden encontrar en la Tabla 5 los resultados estimados de productividad en generación de metros cúbicos de madera y de toneladas de pulpa marrón seca al aire por hectárea.

\begin{tabular}{|c|c|c|c|}
\hline ESPECIE & $\begin{array}{c}\text { Dab } \\
\left(\mathbf{g} / \mathbf{c m}^{3}\right)\end{array}$ & $\mathbf{R B \%}$ & $\begin{array}{c}\text { IMA promedio } \\
\left(\mathbf{m}^{\mathbf{3}} / \mathbf{h a} / \mathbf{a n ̃ o}\right) \\
(* *)\end{array}$ \\
\hline E. grandis (a) & 0.423 & 51.7 & 28 \\
\hline E. maidenii $\mathbf{( b )}$ & 0.559 & 51.4 & 20 \\
\hline E. dunnii (c) & 0.499 & 51.1 & 25 \\
\hline $\begin{array}{c}\text { E. globulus } \\
\text { "jeeralang" (d) }\end{array}$ & 0.550 & 51.4 & 28 \\
\hline
\end{tabular}

$\left.{ }^{* *}\right)$ Valores de IMA promedio de plantaciones comerciales relevadas.

\begin{tabular}{|c|c|c|c|}
\hline $\begin{array}{c}\text { Composición } \\
\text { de Mezcla } \\
(\mathbf{1} / \mathbf{3} \text { por } \\
\text { especie) }\end{array}$ & $\begin{array}{c}\text { Consumo } \\
\text { de mezcla } \\
\left(\mathbf{m}^{3} / \text { ton } \text { a.d. }\right)\end{array}$ & $\begin{array}{c}\text { Productividad } \\
\text { forestal } \\
\text { de mezcla } \\
\left(\mathbf{m}^{3} / \mathbf{h a}\right)\end{array}$ & $\begin{array}{c}\text { Productividad } \\
\text { tecnológica de } \\
\text { mezcla } \\
\text { (ton a.d./ha) }\end{array}$ \\
\hline $\mathbf{a}+\mathbf{b}+\mathbf{c}$ & 3.6 & 219 & $\mathbf{6 1 . 1}$ \\
\hline $\mathbf{b}+\mathbf{c}+\mathbf{d}$ & 3.3 & 219 & $\mathbf{6 6 . 2}$ \\
\hline $\mathbf{a}+\mathbf{c}+\mathbf{d}$ & 3.6 & 243 & $\mathbf{6 7 . 4}$ \\
\hline $\mathbf{a}+\mathbf{b}+\mathbf{d}$ & 3.3 & 219 & $\mathbf{6 8 . 0}$ \\
\hline
\end{tabular}

Tabla 5. Consumos y productividad estimadas de mezclas virtuales de $E$. spp de aproximadamente nueve años.

De acuerdo a este ejercicio, se demuestra que la inserción del $E$. globulus "jeeralang" a una mezcla en cualquiera de las combinaciones, redundaría en una mayor productividad tecnológica, donde se encuentra un aumento promedial del $10 \%$ utilizando cualquier combinación, incluso cuando es elegida $E$. grandis de menor Dab. Esto resulta fundamental, ya que la base de especies alternativas comienza a ser amplia, extendiéndose hacia un gran espectro de materiales que puedan incorporar los pequeños productores de madera para pulpa.

El comparativo propuesto para diferentes especies de eucalipto implantadas en Uruguay induce a pensar que la materia prima disponible presenta una gran diversidad en cuanto a los parámetros más importantes relativos a la performance en procesos kraft. Se encuentra una diferencia notoria en Dab entre especies y entre diferentes plantaciones de una misma especie. Esta diversidad hace interesante la tarea de elección de la o las especies a tener en cuenta para un proyecto de producción de celulosa. La selección depende, en primer lugar, de un componente forestal, donde se deben maximizar propiedades de buen enraizamiento en propagación vegetativa, buena sanidad en vivero, transplante y crecimiento, adaptación al sitio y buen rendimiento de crecimiento por hectárea. En segundo lugar, depende de las propiedades físicas, químicas y anatómicas de la madera, como la densidad aparente básica, contenido de lignina, longitud de fibras y, por último la aptitud en procesos de transformación, como el rendimiento pulpable, blanqueabilidad, propiedades mecánicas y ópticas de la pulpa y papel. 
Como se expresaba en la introducción, el sitio de plantación determina la capacidad de crecimiento y comportamiento forestal de cada especie, siendo notorio en el caso del E. globulus. Buscando especies de eucaliptos con buen comportamiento en el litoral oeste y el norte de Uruguay se encuentran especies como el E. grandis, el $E$. dunnii y el $E$. maidenii que presentan crecimientos acelerados. Queda claro que estas tres especies conforman una buena base forestal con potencial de utilización en una mezcla, dado los resultados similares en requerimientos de álcali activo $(\% \mathrm{AA})$ en cocción kraft. En ese sentido, el E. globulus "jeeralang" también se presenta como una alternativa para ingresar a una mezcla con cualquiera de las anteriores, proporcionando menores consumos de madera.

Finalmente, teniendo en cuenta que tradicionalmente la selección de árboles plus para una propagación vegetativa se ha realizado solamente con objetivos de aumento de productividad forestal, los resultados obtenidos en este trabajo demuestran que es importante incorporar parámetros de selección como el rendimiento pulpable y la densidad aparente básica para obtener buenas productividades tecnológicas. Según lo observado, los parámetros óptimos buscados serían un consumo de $3 \mathrm{~m}^{3} /$ a.d.ton (madera para una tonelada de pulpa seca al aire) y crecimientos acelerados de IMA aproximado a $35 \mathrm{~m}^{3} /$ ha/año. Con estas condiciones se podría llegar a obtener un aumento hasta del $30 \%$ de productividad tecnológica.

\section{Conclusiones}

Se mantiene una diferencia entre el E. globulus comercializado clásicamente por Uruguay y las cuatro especies alternativas más plantadas. Se corrobora el hecho de que las condiciones óptimas para su procesamiento son indudablemente desiguales.

Todas las especies alternativas estudiadas han presentado una gran variabilidad de densidad aparente básica entre diferentes plantaciones y también dentro de cada plantación entre árboles. E. grandis presenta los valores promedio más bajos, afectando el valor de consumo de madera para obtención de celulosa, pero mostrando buenas productividades forestales con crecimientos volumétricos considerables.

Se propone una categorización de las maderas estudiadas en dos franjas. La primera franja desde 12,5 a 15,5\%AA, donde se encuentra el E. globulus y el rango de hasta $0,570 \mathrm{~g} / \mathrm{cm}^{3}$ de E. maidenii. La segunda, desde 15,5 a $19 \%$ AA, en la cual se encuentran el E. grandis, E. dunnii, E. globulus "jeeralang" y E. maidenii (mayor a $0,570 \mathrm{~g} /$ $\left.\mathrm{cm}^{3}\right)$.

Por lo antes expresado E. grandis, E. dunnii, E. maidenii y E. globulus "jeeralang" muestran un gran potencial de utilización en mezclas, dados los resultados similares en requerimientos de álcali activo (\%AA) en cocción kraft, obteniendo rendimientos pulpables brutos promedio cercanos al 51,4 \%.

E. globulus "jeeralang" se posiciona como una alternativa interesante para ingresar a una mezcla con cualquiera de las anteriores, proporcionando menores consumos de madera y una mayor productividad tanto forestal como tecnológica.

Se considera imprescindible incorporar parámetros como el rendimiento pulpable y la densidad aparente básica a los parámetros forestales tradicionales, utilizados en la selección de árboles plus para una propagación vegetativa con el objetivo de obtener buenas productividades tecnológicas.

\section{Referencias}

- BALMELli, G., et al. Potencial del mejoramiento genético para el manejo de enfermedades en Eucalyptus globulus. Montevideo: INIA, 2004. (Serie Técnica; 143).

- DOLDÁN, J. Evaluación de parámetros de calidad de Eucalyptus globulus y Eucalyptus maidenii de plantaciones uruguayas para pulpa de celulosa. Montevideo: LATU, 2007. (Nota Técnica; 8)

- INIA. Seminario Forestal: Mejoramiento genético, silvicultura y sanidad de Eucalyptus globulus en región Sureste: junio de 2002. Tacuarembó: INIA, 2002. (Actividades de Difusión; 289).

- MIRANDA, I.; ALMEIDA, M.H.; PEREIRA, H. Influence of provenance, subspecies, and site on wood density in Eucalyptus Globulus Labill. En: Wood and Fiber Science. 2001, 33(1):9-15.

- RESQUIN, F., et al. Caracterización de la celulosa de especies del género Eucalyptus plantadas en Uruguay. Montevideo: INIA, 2005. (Serie Técnica; 152).

- RIMOLDI, J. A. Consideraciones comerciales del complejo maderero uruguayo. En: Forestal. 2007, (33):9-14.

- URUGUAY. MINISTERIO DE GANADERÍA AGRICULTURA Y PESCA. DIRECCIÓN GENERAL FORESTAL. Boletín estadístico [En línea]. Montevideo: MGAP, 2005. [Consulta: 24 de setiembre de 2008]. Disponible en: http://www.mgap.gub.uy/Forestal/Boletin2005.pdf 